\title{
Die perkutane endoskopische Gastrostomie (PEG)
}

Anika Rosenbaum, Jürgen F. Riemann, Dieter Schilling

Seit ihrer Erstbeschreibung im Jahr 1980 [1] hat sich die perkutane endoskopische Gastrostomie nach dem Fadendurchzugsverfahren zu einem Standardverfahren für die längerfristige enterale Ernährung entwickelt. Wann eine PEG indiziert ist, auf welche Kontraindikationen Sie achten müssen und wie die Sonde richtig angelegt wird, zeigt Ihnen unsere Schritt-für-Schritt-Anleitung.

\section{Indikationen}

Dauer der enteralen Ernährung I Über die perkutane endoskopische Gastrostomie (PEG) sollten Patienten ernährt werden, die aufgrund ihrer Grunderkrankung ( $\triangleright$ Tab. 1) längerfristig keine oder nur unzureichende Mengen Nahrung auf dem oralen Weg zu sich nehmen können.

Eine PEG ist indiziert, wenn der Patient für mehr als 4-6 Wochen enteral ernährt werden muss [2].

Auch wenn eine Ösophagitis als Nebenwirkung einer Radiatio zu erwarten ist, kann eine prophylaktische PEG-Anlage gerechtfertigt sein. Eine weitere Spezialindikation ist die Ableitungs-PEG bei ausgeprägter Peritonealkarzinose mit unstillbarem Erbrechen. Bei Magenausgangsstenose, Magenmotilitätsstörung oder Zustand nach Gastrektomie kann in analoger Technik die perkutane endoskopische Jejunostomie (PEJ) angelegt werden. Alternativ kann man über eine vorhandene gastrale PEG endoskopisch eine Jejunalsonde einlegen (Jet-PEG).

PEG am Lebensende I Die künstliche Ernährung am Lebensende (z.B. bei fortgeschrittener Demenzerkankung) wurde über längere Zeit heftig diskutiert und wird inzwischen nur noch in

Tab.1 Mögliche Indikationen für eine PEG-Anlage.

Neurogene Schluckstörungen

Mechanisch bedingte Schluckstörungen (z. B. durch Tumore, Narben)

Zu erwartende Ösophagitis unter Radiatio

Geriatrische Patienten (Demenz im Frühstadium mit mangelnder Nahrungsaufnahme)

Intensivpatienten (sobald längerfristige enterale Ernährung absehbar ist)

Kurzdarmsyndrom (bei Rest-Dünndarm> $100 \mathrm{~cm}$, sonst TPE)

Inappetenz

Ausgeprägte Malnutrition, Kachexie
Ausnahmefällen als sinnvoll angesehen. Die PEGSonde kann niemals ein Ersatz für menschliche und pflegerische Zuwendung sein.

\section{Kontraindikationen}

Absolute Kontraindikationen I Eine PEG sollten Sie nicht anlegen bei:

- einer Lebenserwartung $<4$ Wochen

- fehlendem endoskopischen Zugang

- schweren Gerinnungsstörungen (Quick $<45 \%$, Thrombozyten $<50000 / \mu \mathrm{l}$ )

- Magenausgangsstenose oder Magenentleerungsstörung (PEJ oder Jet-PEG wählen)

- einer Akutphase einer Erkrankung

- Schockgeschehen

- akuten Stoffwechselentgleisungen

- Perforation des Gastrointestinaltrakts

- Peritonitis

Relative Kontraindikationen I $\mathrm{Zu}$ den relativen Kontraindikationen zählen:

- fehlende Diaphanoskopie (s.u.)

- größere Menge Aszites

- Ulkusleiden oder Tumor (keine Punktion im Bereich der Läsion)

- gestörte Darmpassage (z.B. Ileus, Peritonealkarzinose)

- Malabsorption

- sehr unruhige Patienten, die sich durch Zug an der Sonde verletzen könnten

- geplanter Magenhochzug bei Ösophaguskarzinom (mit dem Chirurgen absprechen, ggf. parenterale Ernährung oder PEJ wählen)

\section{Bevor es losgeht}

Patientenaufklärung I Die Aufklärung des Patienten - bzw. die des Betreuers bei nicht geschäftsfähigen Patienten - sollte mindestens 24 Stunden vor dem Eingriff stattfinden. Eine schriftliche Einwilligung muss vorliegen. Im Aufklärungsgespräch sollten Sie über leichte und schwere Akutkomplikationen ( $\downarrow$ Tab. 2 ) informieren, sowie über die adäquate Nachsorge, um 


\begin{tabular}{|c|c|c|}
\hline & Akut & Chronisch \\
\hline \multirow[t]{2}{*}{ Leicht } & Wundschmerzen, Pneumoperitoneum, Lokalinfektion & Sondenprobleme, Leckagen \\
\hline & Hautemphysem & Granulationen \\
\hline \multirow[t]{4}{*}{ Schwer } & $\begin{array}{l}\text { Perforation oder Verletzung angrenzender Organe } \\
\text { (z.B. Colon transversum, Leber, Dünndarm, Milz, Aorta) }\end{array}$ & Einwachsen der inneren Haltplatte (Buried Bumper) \\
\hline & Blutung & Sondendislokation \\
\hline & Fistelbildung & Chronische Infektion \\
\hline & Peritonitis & Aspiration \\
\hline
\end{tabular}

Tab.2 Komplikationen der PEG-Anlage.

Bei allen Patienten, die ein erhöhtes Risiko für eine MRSA-Infektion aufweisen, wird vor PEG-Anlage ein MRSA-Screening empfohlen mit nachfolgender Dekontamination bei positivem Nachweis [5].

Aszites ausschließen | Ein aktueller Gerinnungsstatus muss vorliegen. Wir führen zudem routinemäßig einen Ultraschall durch, um größere Mengen Aszites auszuschließen. Der Patient sollte mindestens 8 Stunden nüchtern sein und einen intravenösen Zugang für die Sedierung erhalten. Falls nötig, wird die Bauchdecke rasiert.

Antibiotikaprophylaxe I Etwa 30 Minuten vor dem Eingriff wird eine einmalige Antibiotikaprophylaxe mit einem 2.-Generations-Cephalosporin (z.B. 2 g Cefazolin i.v. oder 1,5 g Cefuroxim i.v.) verabreicht, um lokale Infektionen zu verhindern [3, 4]. Patienten, die bereits aus anderen Gründen ein Breitspektrum-Antibiotikum erhalten, benötigen keine zusätzliche Prophylaxe.

\section{So wird's gemacht: PEG}

Benötigte Materialien | Die PEG-Anlage wird von einem Endoskopie-Team ausgeführt - zusammen mit einem zweiten Arzt, der die Punktion übernimmt. Für den Eingriff benötigt man ( Abb. 1):

- eine Spritze mit Lokalanästhetikum (5-10 ml)

- sterile Kompressen

- eine Schlitzkompresse

- Abdecktücher

- mit Desinfektionsmittel getränkte Tupfer

- eine Zange

Abb.1 Materialien für die PEG: (1) steriler Kittel, (2) Lochtuch, (3) sterile Handschuhe, (4) Abdecktücher, (5) Spritze für das Lokalanästhetikum (5-10 ml), (6) Zange, (7) mit Desinfektionsmittel getränkte Tupfer, (8) sterile Kompressen, (9) Schlitzkompresse. Nicht im Bild: Haube und Mundschutz.

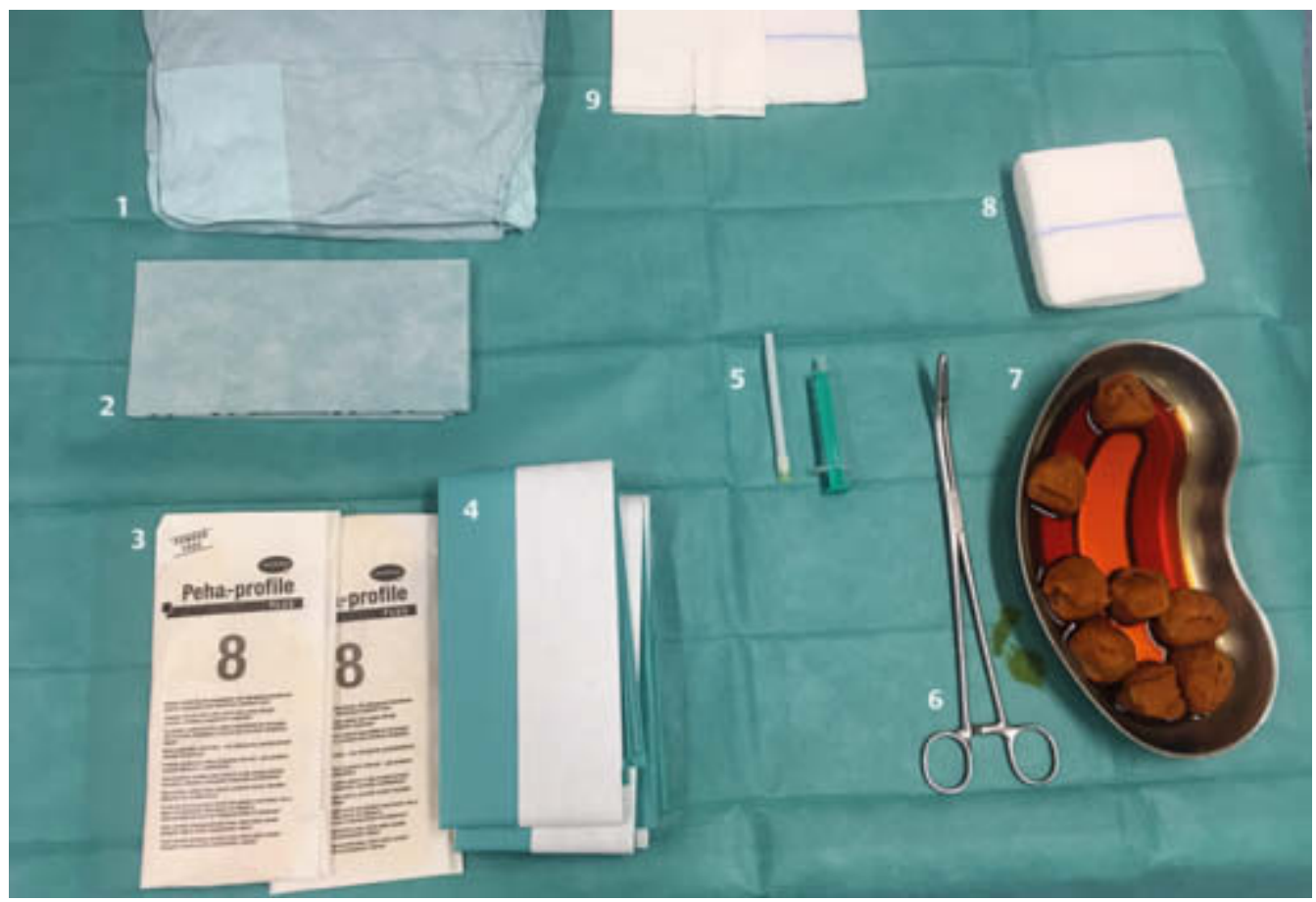

Abb.2 PEG-Anlage-Set mit Skalpell und Trokar. 


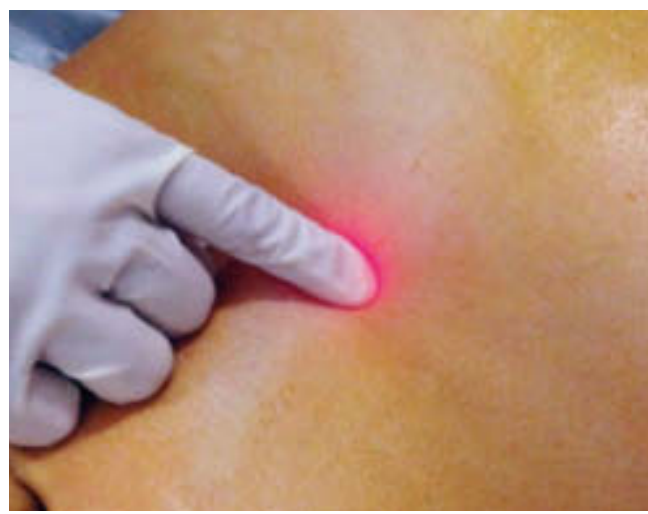

Abb. 3 Mit Hilfe der Diaphanoskopie wird nach einer geeigneten Punktionsstelle gesucht.

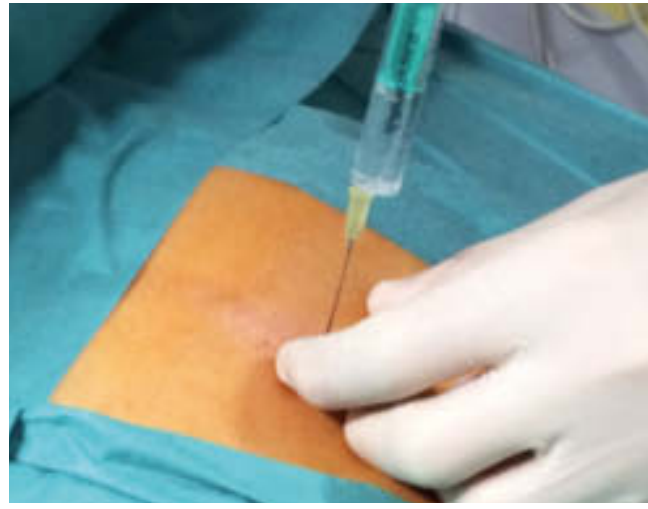

Abb. 5 Nadelaspirationstest mit gefüllter Spritze (Lokalanästhetikum oder Kochsalz).

Weiterhin sollten bereitliegen:

- Endoskopieeinheit

- PEG-Anlageset mit Skalpell ( $>$ Abb. 2)

- Punktionsnadel mit Mandrin

- Faden

- PEG-Sonde (15 oder $20 \mathrm{CH}$ ) mit Halteplatte und Adaptern

Der Assistent führt den Eingriff unter sterilen Bedingungen nach chirurgischem Standard mit Haube, Mundschutz, Kittel und sterilen Handschuhen durch.

Sedierung und Desinfektion I Der Patienten liegt in Rückenlage - abgesehen davon wird der Eingriff wie eine Gastroskopie vorbereitet. In der Regel wird mit Propofol fraktioniert i.v. sediert. Während der Endoskopiker das Gerät einführt, um mit Hilfe einer Ösophago-Gastro-Duodenoskopie Kontraindikationen auszuschließen, deckt der Assistent steril ab und desinfiziert großflächig die Bauchdecke.

Punktionsstelle finden | Mit dem Endoskop wird eine geeignete Stelle an der Magenvorderwand aufgesucht - typischerweise am Übergang vom Antrum zum Corpus. Im abgedunkelten Raum wird geprüft, ob dort der Lichtschein des Endoskops durch die Bauchdecke dringt (Diaphanosko-

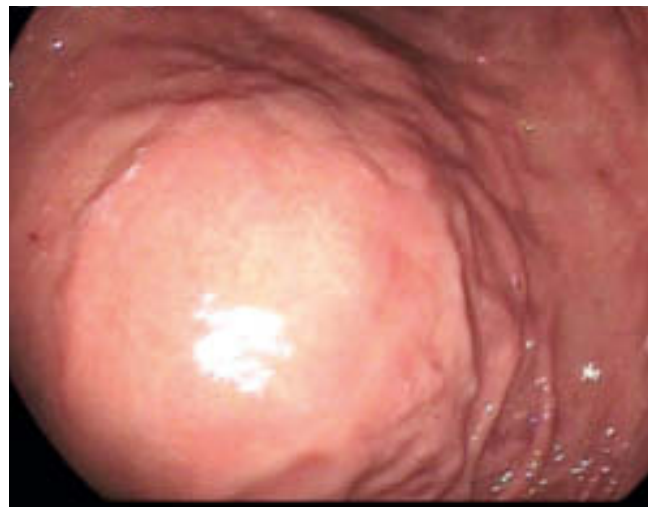

Abb. 4 Durch Druck von außen auf die vom Endoskop beleuchtete Stelle der Bauchdecke wird die mögliche Punktionsstelle von innen überprüft.

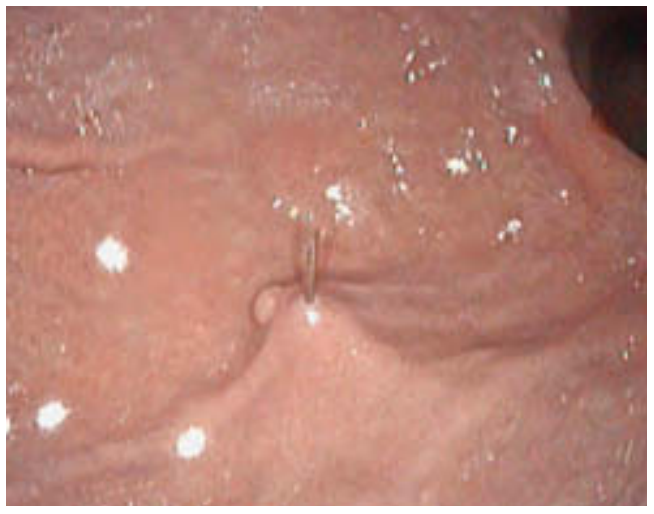

Abb. 6 Die Nadel ist im Magenlumen sichtbar und wird zur Markierung belassen, bis eine Stichinzision gesetzt ist.

pie). Dies zeigt an, dass an dieser Stelle der Magen der Bauchdecke direkt anliegt. Durch Fingerdruck von außen ( $\bullet$ Abb. 3 ) überprüft man die Position der möglichen Punktionsstelle von innen ( Abb. 4).

Punktion | Nach lokaler Anästhesie der Haut und Bauchdecke wird unter Aspiration der Magen punktiert. Bei schwacher Diaphanoskopie ist ein Nadelaspirationstest mit einer kochsalzgefüllten Spritze sinnvoll ( Abb. 5). Hierbei muss das Magenlumen ohne vorherige Luftaspiration erreichbar sein. Ist Luft aspirabel und gleichzeitig die Nadel im Magenlumen sichtbar ( $\bullet$ Abb. 6), wird die Spritze entfernt, die Nadel zur Markierung belassen und dort eine kleine Stichinzision (ca. $5 \mathrm{~mm}$ ) gesetzt. Durch diese wird in gleicher Stichrichtung wie mit der Anästhesienadel die Punktionsnadel eingeführt ( $\mathbf{A}$ bb. 7 und 8). Nach Entfernen des Mandrins wird der doppelt liegende Faden durch die Hülse in das Magenlumen vorgeschoben $(\bullet$ Abb. 9).

Nach Entfernen des Mandrins sollten Sie rasch die Punktionsnadel mit dem Finger abdichten. Sonst entweicht Luft aus dem Magen, was die Endoskopie erschwert. 


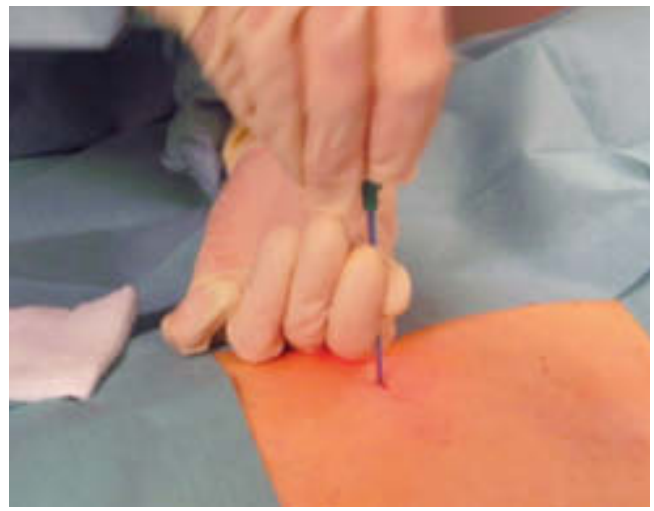

Abb. 7 Durch die Stichinzision wird die Punktionsnadel eingeführt.

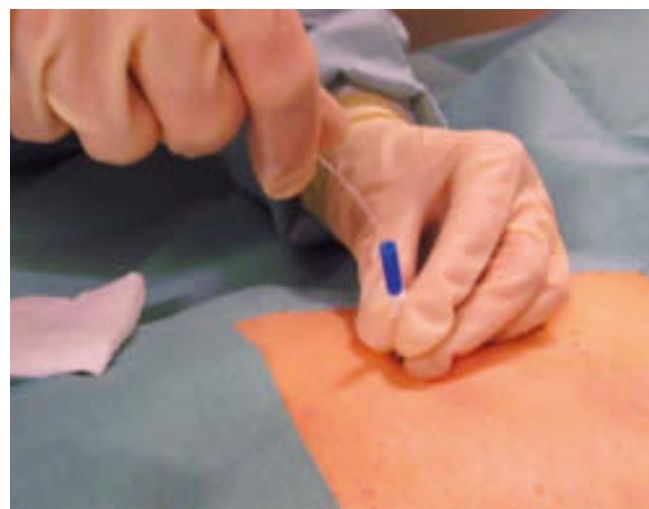

Abb. 9 Nach Entfernen des Mandrins wird ein Faden durch die Hülse in das Magenlumen vorgeschoben.

Durchziehen des Fadens I Während der Faden durch die Hülse in den Magen gelangt, wird eine Fasszange in das Endoskop eingeführt. Mit dieser wird der Faden gefasst, die geschlossene Zange ein kleines Stück ins Endoskop eingezogen und das Gerät dann langsam entfernt. Der Faden wird so aus dem Mund des Patienten hinausgezogen und die PEG-Sonde daran befestigt ( $\triangleright$ Abb. 10). Nun zieht der Assistent den Faden mit der Sonde durch Mund und Ösophagus in den Magen (Abb. 11 und 12), bis die innere Halteplatte der Sonde der Magenwand anliegt ( Abb. 13).

Wenn Sie beim Herausziehen einen leichten Widerstand spüren, liegt die innere Halteplatte korrekt an.

Zuletzt wird der Konus der Sonde außen abgeschnitten, die äußere Halteplatte, die Klemme und das Ansatzstück werden über die Sonde geschoben und befestigt. Die äußere Haltplatte wird über einer Schlitzkompresse zunächst für 12 bis maximal 24 Stunden unter ganz leichtem Zug ohne Spannung adaptiert ( Abb. 14).

Komplikationen | Komplikationen der PEG-Anlage sind vielfältig und können akut oder im

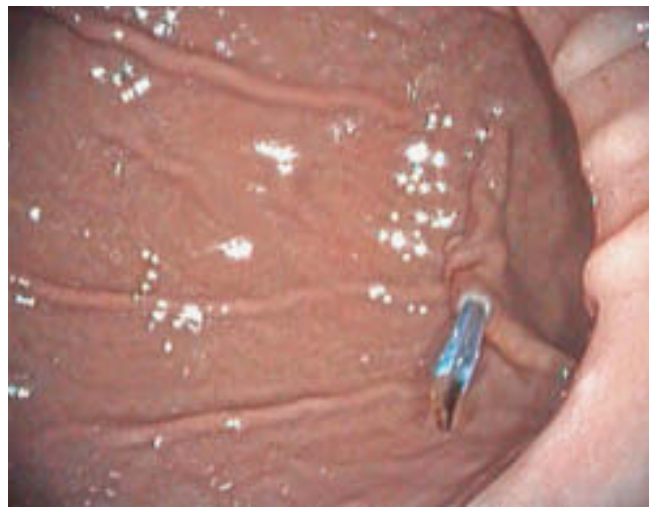

Abb. 8 Ansicht von innen: Die Stichrichtung der Punktionsnadel ist die gleiche wie die der Anästhesienadel.

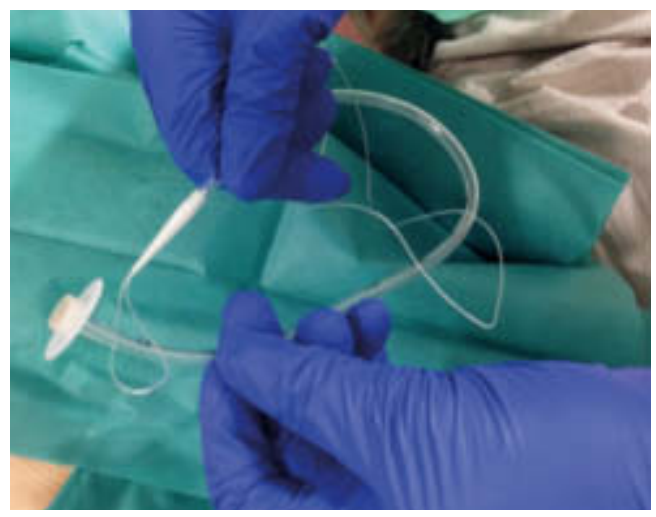

Abb. 10 Die PEG-Sonde wird an dem Faden befestigt.

weiteren Verlauf auftreten ( $\triangleright$ Tab. 2). In 30\% der Fälle kommt es zu lokalen Infektionen - die häufigste Komplikation der PEG. Schwere Komplikationen treten bei 0,4 bis 4,4\% der Prozeduren auf und erfordern eine weitere Intervention. Dazu gehören:

- peristomale Leckage mit Peritonitis

- Nachblutung

- Verletzung angrenzender Organe

- Fistelbildung

- Tod $[6,7]$

\section{Nachsorge}

Kostaufbau | 4-6 Stunden nach dem Eingriff können Flüssigkeit und Sondenkost mit langsamer Flussrate über die PEG gegeben werden. Der Kostaufbau folgt dann in der Regel einem Plan über mehrere Tage.

Verbandswechsel I Nach 12-24 Stunden muss der Verband zum ersten Mal gewechselt und die äußere Halteplatte gelockert werden. Nach Desinfektion mit einem alkoholhaltigen Hautantiseptikum wird die Sonde um $360^{\circ}$ gedreht und $4-5 \mathrm{~cm}$ in den Magen hinein mobilisiert. Anschließend zieht man sie wieder zurück (bis man 


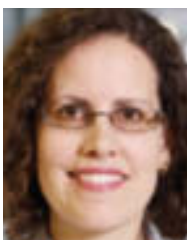

Dr. med. Anika Rosenbaum ist Oberärztin an der Medizinischen Klinik II am Diakonissenkrankenhaus Mannheim. anika.rosenbaum@ diakonissen.de

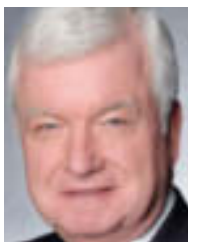

Prof. Dr. med. Jürgen F. Riemann ist ehem. Direktor der Medizinischen Klinik C am Klinikum Ludwigshafen. riemannj@garps.de

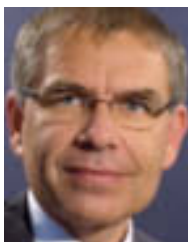

Prof. Dr. med.

Dieter Schilling

ist Chefarzt der Medizinischen Klinik II am Diakonissenkrankenhaus Mannheim. dieter.schilling@diakonissen. de

\section{Interessenkonflikt}

AR und DS geben an,

Referentenhonorare von der Firma Fresenius Kabi erhalten zu haben.

JFR gibt an, dass kein Interessenkonflikt besteht.

DOI 10.1055/s-0041-103316

Dtsch Med Wochenschr 2015; 140: 1072-1076

(c) Georg Thieme Verlag KG . Stuttgart · New York . ISSN 0012-0472

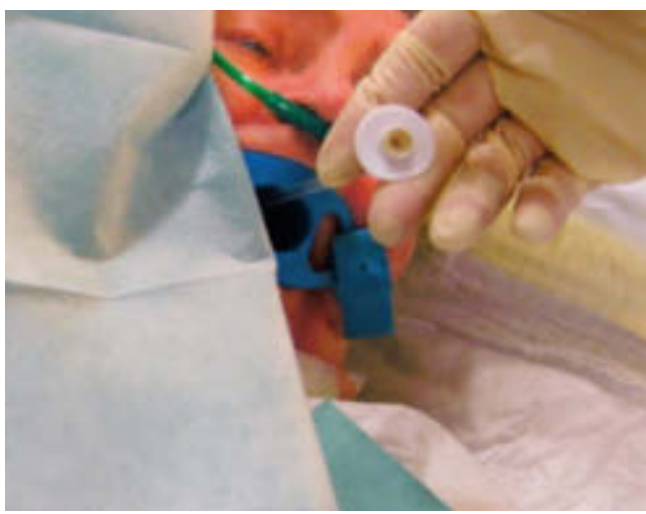

Abb. 11 Die PEG-Sonde wird durch Mund und Ösophagus in den Magen hinein ...

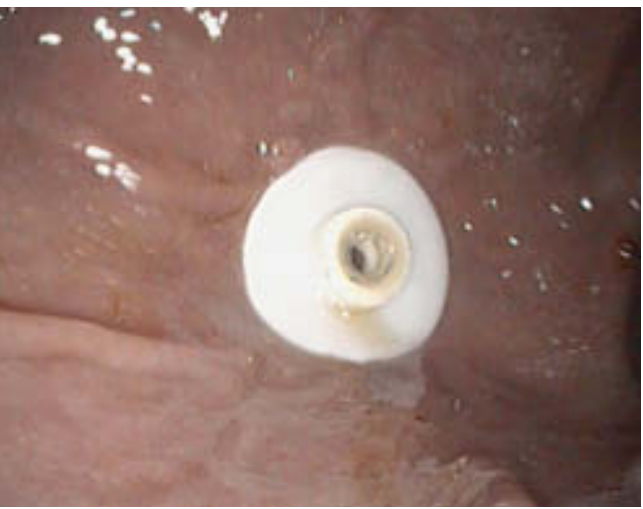

Abb. 13 ... bis die innere Halteplatte korrekt an der Magenwand anliegt.

einen leichten Widerstand spürt) und fixiert die äußere Halteplatte ca. $5 \mathrm{~mm}$ über der Bauchdecke. Bis sich ein stabiler Stomakanal gebildet hat (nach ca. 8 Tagen) sollte man den Verband täglich wechseln. Danach reicht ein Wechsel 2-3 mal pro Woche.

Die Sonde muss bei jedem Verbandswechsel mobilisiert werden, um zu verhindern, dass die inneren Halteplatte einwächst („Buried Bumper").

\section{Wichtiges in Kürze}

- Eine PEG ist indiziert, wenn der Patient für mehr als 4-6 Wochen enteral ernährt werden muss.

- Bei Patienten mit erhöhtem Risiko für eine MRSA-Infektion sollte vor der PEG-Anlage ein MRSA-Screening durchgeführt werden.

- Der Eingriff erfolgt unter sterilen Bedingungen.

- Die häufigsten Komplikationen der PEG sind lokale Infektionen.

- Die Sonde muss bei jedem Verbandswechsel mobilisiert werden, um zu verhindern, dass die inneren Halteplatte einwächst („Buried Bumper“).

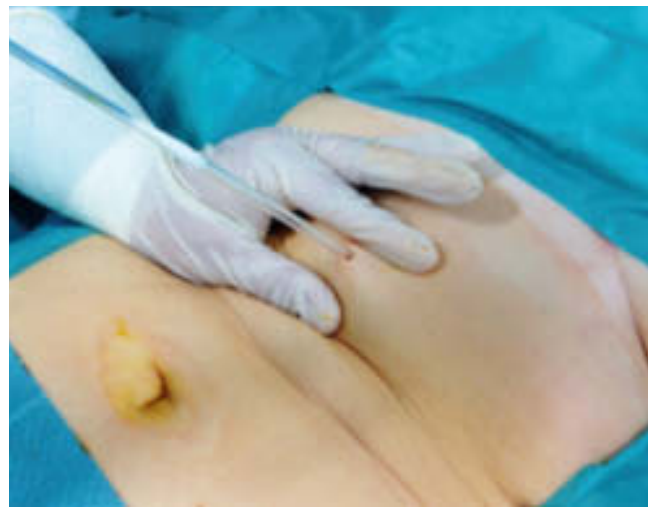

Abb. $12 \ldots$ und an der Bauchwand herausgezogen, ...

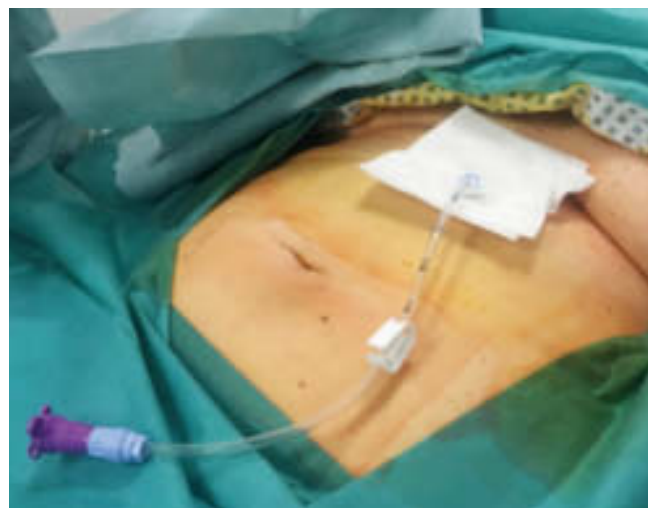

Abb. 14 Die äußere Haltplatte wird für 12-24h unter ganz leichtem Zug ohne Spannung adaptiert.

\section{Literatur}

1 Gauderer MW, Ponsky JL, Izant RJ Jr. Gastrostomy without laparotomy: a percutaneous endoscopic technique. J Pediatr Surg 1980; 15: 872-875

2 ESPEN Guidelines on Enteral Nutrition. Clinical Nutrition 2006; 25: 260-274

3 Sharma VK, Howden CW. Meta-analysis of randomized, controlled trials of antibiotic prophylaxis before percutaneous endoscopic gastrostomy. Am J Gastroenterol 2000; 95 : 3133-3136

4 Lipp A, Lusardi G. Systemic antimicrobial prophylaxis for percutaneous endoscopic gastrostomy. Cochrane Database Syst Rev. 2013; DOI: 10.1002/14651858.CD005571.pub3

5 Robert Koch Institut. Empfehlungen zur Prävention und Kontrolle von MRSA-Stämmen in Krankenhäusern und anderen medizinischen Einrichtungen (6/14). http://www.rki.de/DE/Content/Infekt/ Krankenhaushygiene/Kommission/Downloads/ MRSA_Rili.html; Stand: 22.02.2008

6 Schapiro GD, Edmundowicz SA. Complications of percutaneous endoscopic gastrostomy. Gastrointest Endosc Clin N Am 1996; 6: 409-422

7 Teichmann J, Riemann JF. Techniques and complications in post-interventional and long term enteral nutrition. Internist 2007; 48: 1076-1083 